\title{
Magnetocrystalline anisotropy of Fe and Co slabs and clusters on $\mathrm{SrTiO} 3$ by first- principles
}

\section{Li, Dongzhe; Barreteau, Cyrille; Smogunov, Alexander}

\section{Published in:}

Physical Review B

Link to article, DOI:

10.1103/PhysRevB.93.144405

Publication date:

2016

Document Version

Publisher's PDF, also known as Version of record

Link back to DTU Orbit

Citation (APA):

Li, D., Barreteau, C., \& Smogunov, A. (2016). Magnetocrystalline anisotropy of Fe and Co slabs and clusters on $\mathrm{SrTiO}_{3}$ by first-principles. Physical Review B, 93(14), [144405]. https://doi.org/10.1103/PhysRevB.93.144405

\section{General rights}

Copyright and moral rights for the publications made accessible in the public portal are retained by the authors and/or other copyright owners and it is a condition of accessing publications that users recognise and abide by the legal requirements associated with these rights.

- Users may download and print one copy of any publication from the public portal for the purpose of private study or research.

- You may not further distribute the material or use it for any profit-making activity or commercial gain

- You may freely distribute the URL identifying the publication in the public portal 


\title{
Magnetocrystalline anisotropy of $\mathrm{Fe}$ and $\mathrm{Co}$ slabs and clusters on $\mathrm{SrTiO}_{3}$ by first-principles
}

\author{
Dongzhe Li, ${ }^{1}$ Cyrille Barreteau, ${ }^{1,2}$ and Alexander Smogunov ${ }^{1, *}$ \\ ${ }^{1}$ Service de Physique de l'Etat Condensé (SPEC), CEA, CNRS, Université Paris-Saclay, CEA Saclay 91191 Gif-sur-Yvette Cedex, France \\ ${ }^{2}$ DTU NANOTECH, Technical University of Denmark, Ørsteds Plads 344, DK-2800 Kgs. Lyngby, Denmark
}

(Received 9 February 2016; published 5 April 2016)

\begin{abstract}
In this paper, we present a detailed theoretical investigation of the electronic and magnetic properties of ferromagnetic slabs and clusters deposited on $\mathrm{SrTiO}_{3}$ via first-principles calculations, with a particular emphasis on the magnetocrystalline anisotropy (MCA). We found that in the case of Fe ultrathin films deposited on $\mathrm{SrTiO}_{3}$ the effect of the interface is to quench the MCA whereas for Co we observe a spin reorientation from in-plane to out-of-plane as compared to the free surface. We also find a strong enhancement of MCA for small clusters upon deposition on $\mathrm{SrTiO}_{3}$ substrate. The origin of this enhancement of MCA is attributed to the hybridization between the substrate and the $d$ orbitals of the cluster extending in-plane for Fe and out-of-plane for Co. As a consequence, we predict that the Fe nanocrystals (even rather small) should be magnetically stable and are thus good potential candidates for magnetic storage devices.
\end{abstract}

DOI: 10.1103/PhysRevB.93.144405

\section{INTRODUCTION}

The fine tuning of the interfacial magnetocrystalline anisotropy (MCA) in ferromagnet-oxide insulator systems represents a key issue for several technological applications such as perpendicular magnetic tunnel junctions ( $p$-MTJs) [1-3] and tunneling anisotropic magnetoresistive (TAMR) systems [4,5]. It is well known that the physical origin of the MCA is the spin-orbit coupling (SOC). For the $3 d$ transition metals the SOC being of the order of a few tens of meV, the MCA per atom is extremely small $\left(10^{-3} \mathrm{meV}\right)$ in the bulk phase of cubic materials but can get larger $\left(\sim 10^{-1} \mathrm{meV}\right)$ at surfaces/interfaces due to reduced symmetry. In order to obtain even larger MCA, traditionally, the MCA of nanostructures of $3 d$ elements is enhanced by introducing $4 d$ or $5 d$ heavy elements with large SOC as a substrate such as $\mathrm{Co} / \mathrm{Pt}$ [6] or $\mathrm{Co} / \mathrm{Pd}$ [7] multilayers as well as in small $3 d$ clusters on heavy elements substrate [8]. However, despite the weak SOC at the interface, a strong MCA has been observed in CoFe thin films on metallic oxides such as $\mathrm{AlO}_{x}$ [9] and $\mathrm{MgO}$ [10]. H. X. Yang et al. reported a very large perpendicular magnetic anisotropy at ferromagnetic $\mid \mathrm{MgO}$ interface due to strong hybridization between the transition metal $3 d$ orbitals and $\mathrm{O}-2 p_{z}$ orbital [11]. More recently, Ran et al. have shown that it was possible to reach the magnetic anisotropy limit $(\sim 60 \mathrm{meV})$ of $3 d$ metal atom by coordinating a single $\mathrm{Co}$ atom to the $\mathrm{O}$ site of an $\mathrm{MgO}$ surface [12]. Enhancing MCA of nanostructures provides a route towards future miniaturization of data storage at ultimate length scales $[13,14]$

In our previous work, we demonstrated that for both truncated-pyramid shaped Fe and Co nanocrystals, the MCA of free nanocrystals is mainly dominated by the basal (001) facets resulting in an opposite behavior: out-of-plane and in-plane magnetization direction favored in Fe and Co nanocrystals, respectively $[15,16]$. Therefore, the study of magnetic properties of nanocrystals deposited on a $\mathrm{SrTiO}_{3}$ as experimentally obtainable [16,17], is essential, since a considerable influence on the overall behavior of the nanocrystals is expected due to

*alexander.smogunov@cea.fr the bonding between the substrate and basal (001) facet. In this paper, we report first-principles investigations of the MCA of bcc-Fe(001) and fcc-Co(001) deposited on a $\mathrm{SrTiO}_{3}$ substrate, namely $\mathrm{Fe}(\mathrm{Co}) \mid \mathrm{SrTiO}_{3}$ interface. Next, we also investigated the MCA of very small (five atoms) $\mathrm{Fe}$ and Co clusters on $\mathrm{SrTiO}_{3}$, namely $\mathrm{Fe}_{5}\left(\mathrm{Co}_{5}\right)-\mathrm{SrTiO}_{3}$

The paper is organized as follows. In Sec. II, we present the computational method used in this work. In Sec. III, the electronic and magnetic properties of $\mathrm{Fe}(\mathrm{Co}) \mid \mathrm{SrTiO}_{3}$ interface followed by the small $\mathrm{Fe}$ and $\mathrm{Co}$ deposited on a $\mathrm{SrTiO}_{3}$ substrate. Finally, the conclusion will be drawn in Sec. IV.

\section{CAlCulation method}

We carried out the first-principles calculations by using the plane wave electronic structure package QUANTUM ESPRESSO (QE) [18]. Generalized gradient approximation in Perdew, Burke, and Ernzerhof parametrization [19] was used for electronic exchange-correlation functionals and a plane wave basis set with the cutoffs of $30 \mathrm{Ry}$ and $300 \mathrm{Ry}$ were employed for the wave functions and for the charge density, respectively. The MCA was calculated from the band energy difference between two magnetic orientation $\hat{\mathbf{m}}_{1}$ and $\hat{\mathbf{m}}_{2}$ using force theorem [16], as we implemented recently in QE package:

$$
\mathrm{MCA}=\sum_{\alpha \mathrm{occ}} \epsilon_{\alpha}\left(\hat{\mathbf{m}}_{1}\right)-\sum_{\alpha \mathrm{occ}} \epsilon_{i}\left(\hat{\mathbf{m}}_{2}\right)
$$

where $\epsilon_{\alpha}(\hat{\mathbf{m}})$ are the eigenvalues obtained after a single diagonalization of the Hamiltonian including SOC, but starting from an initial well converged charge/spin magnetization density of a self-consistent scalar-relativistic calculation that has been rotated to the appropriate spin orientation axis as explained in Ref. [16].

The $\mathrm{Fe}(\mathrm{Co}) \mid \mathrm{SrTiO}_{3}$ interface was simulated by 10 layers of bcc-Fe(001)[fcc-Co(001)] slab deposited on $\mathrm{a} \mathrm{SrTiO}_{3}(001)$ terminated by $\mathrm{TiO}_{2}$ with five layers. In the ionic relaxation, the Brillouin zone has been discretized by using $10 \times 10$ in-plane $k$-points mesh and a smearing parameter of $10 \mathrm{mRy}$. Two bottom layers of $\mathrm{SrTiO}_{3}$ were fixed while other three layers of substrate and ferromagnetic slabs were relaxed until the atomic 
forces are less than $1 \mathrm{meV} / \AA$. We used $15 \AA$ of vacuum space in the $z$ direction in order to avoid the unphysical interactions between two adjacent elementary unit cells. To obtain reliable values of MCA, the convergence of calculations has been carefully checked. A mesh of $20 \times 20$ in-plane $k$ points has been used for SCF calculation with scalar-relativistic PPs with a smaller smearing parameter of $5 \mathrm{mRy}$. In non-SCF calculation with full-relativistic PPs including SOC the mesh was increased to $60 \times 60$ and smearing parameter was reduced down to $1 \mathrm{mRy}$ which provides an accuracy of MCA below $10^{-2} \mathrm{meV}$.

For small Fe and Co clusters on $\mathrm{SrTiO}_{3}$, the interface was simulated by a $(4 \times 4)$ in-plane $\mathrm{TiO}_{2}$-terminated $\mathrm{SrTiO}_{3}(001)$ substrate with five atomic layers containing one $\mathrm{Fe}(\mathrm{Co})$ cluster made of five atoms. Two bottom layers were fixed while other three layers of substrate and $\mathrm{Fe}(\mathrm{Co})$ cluster were relaxed until atomic forces are less than $1 \mathrm{meV} / \AA$. For both scalar and full relativistic calculations, a $(8 \times 8 \times 1) k$-points mesh and a smearing parameter of $1 \mathrm{mRy}$ was used. In addition, the effect of unphysical interaction in the direction $z$ was minimized by taking a vacuum space of about $15 \AA$.

\section{RESULTS AND DISCUSSIONS}

\section{A. $\mathrm{Fe}(\mathrm{Co}) \mid \mathrm{SrTiO}_{3}$ interfaces}

The $\mathrm{SrO}$ and $\mathrm{TiO}_{2}$ planes in the perovskite cubic $\mathrm{SrTiO}_{3}$ alternate in the (001) direction, here $\mathrm{SrTiO}_{3}(001)$ surface was chosen to be $\mathrm{TiO}_{2}$ terminated since it is energetically more favorable than the $\mathrm{SrO}$ terminated one [20]. The lattice constants of bulk bcc-Fe, fcc-Co, and $\mathrm{SrTiO}_{3}$ are 2.85, 3.53, and $3.93 \AA$, as compared to the experimental values of 2.87 , 3.54, and $3.91 \AA$. When deposited on $\mathrm{SrTiO}_{3}$ the in-plane lattice parameter of $\mathrm{Fe}(\mathrm{Co})$ slab is imposed by the one of bulk $\mathrm{SrTiO}_{3}$ since it has been shown that the Co layer can nicely be grown on this substrate [21,22]. In order to obtain a better match, the $\mathrm{Fe}$ and Co slabs are rotated by $45^{\circ}$ with respect to the substrate, and each layer of the ferromagnetic slab is made of 2 atoms per supercell. The $\mathrm{TiO}_{2}$ layer at the interface in $\mathrm{Fe}(\mathrm{Co}) \mid \mathrm{SrTiO}_{3}$ is denoted as $\mathbf{S}$ (see Fig. 1). Layers toward the $\mathrm{SrTiO}_{3}$ bulk are labeled as $\mathbf{S}-1, \mathbf{S}-2$, etc., while $\mathrm{Fe}(\mathrm{Co})$ layers towards the surface are labeled as $\mathbf{S}+1, \mathbf{S}+2, \mathbf{S}+3$, etc.

We found that the most stable configuration is, in all cases, where the $\mathrm{Fe}(\mathrm{Co})$ sites in layer $\mathbf{S}+1$ are on top of the $\mathrm{O}$ sites in layer $\mathbf{S}$ with the distance of 1.961(1.968) $\AA$. This is in agreement with previous study in Ref. [23]. The mismatch with $\mathrm{SrTiO}_{3}$ was found to be about -2.5 and $10.1 \%$ for $\mathrm{Fe}$ and $\mathrm{Co}$, respectively. The $\mathrm{Fe}$ and Co slabs have been strained and relaxed to accommodate the lattice structure of the $\mathrm{SrTiO}_{3}$ substrate, respectively. As a result, one finds that the distances beween $\mathbf{S}$ and $\mathbf{S}+1$ of about $1.501 \AA$ and $1.378 \AA$ which should be compared with the bulk values of $1.425 \AA$ and $1.765 \AA$ for Fe and Co, respectively.

\section{Magnetic spin moment}

We plot in Fig. 2 the local spin moments of a free $\mathrm{Fe}(\mathrm{Co})$ slab (blue circles) but for which the ionic positions are the one obtained after relaxation in presence of the $\mathrm{SrTiO}_{3}(001)$ substrate. In this way we can evaluate the role of the relaxation

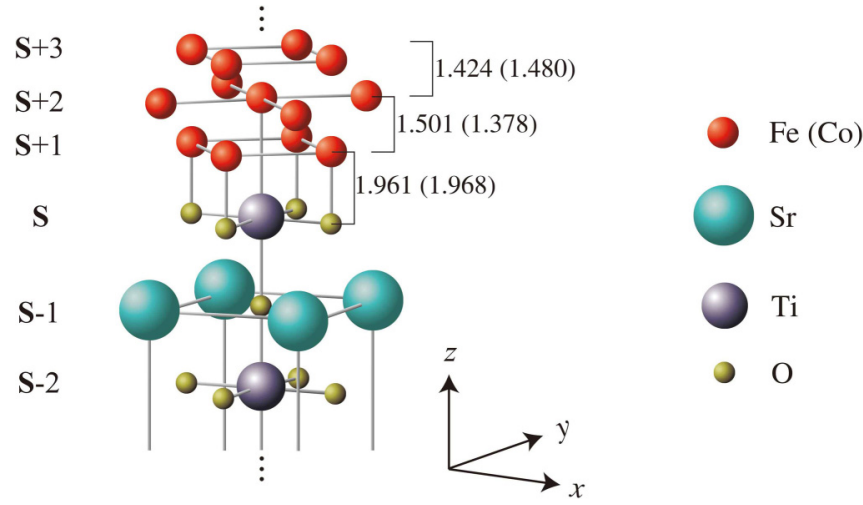

FIG. 1. Atomic structure of bcc-Fe(001) and fcc-Co(001) slabs on top of $\mathrm{TiO}_{2}$-terminated (001) surface of $\mathrm{SrTiO}_{3}$. The ferromagnetic slab is rotated by $45^{\circ}$ with respect to substrate in order to better match with the $\mathrm{SrTiO}_{3}$ lattice. Note that each layer of ferromagnetic slabs is made of 2 atoms per supercell. Layers $\mathbf{S}+3, \ldots, \mathbf{S}-2$ are shown and the distances in the $z$ direction between different ferromagnetic layers are also indicated.

on the free surface as compared to the interface. The local spin moments of the full system $\mathrm{Fe}(\mathrm{Co}) \mid \mathrm{SrTiO}_{3}(001)$ are shown in red squares. For free slabs, the magnetic moment of $\mathbf{S}+1$ layer are enhanced up to 3.07 and $1.97 \mu_{\mathrm{B}}$ with respect to their bulk values of 2.15 and $1.79 \mu_{\mathrm{B}}$ in $\mathbf{S}+5$ layer for $\mathrm{Fe}$ and $\mathrm{Co}$, respectively. However, in the case of $\mathrm{Fe}(\mathrm{Co}) \mid \mathrm{SrTiO}_{3}$, the surface spin moment is reduced to 2.61 and $1.74 \mu_{\mathrm{B}}$ (it is even smaller than its bulk value) due to bonding and charge transfer at the interface. In addition, the hybridization between $\mathrm{Fe} 3 d$ and states of $\mathrm{TiO}_{2}$ at the interface induces spin moments on $\mathrm{Ti}$ and $\mathrm{O}$ atoms. It has been found that the induced magnetic moment of the interface $\mathrm{O}$ atom in $\mathbf{S}$ layer is $\sim 0.05(0.06) \mu_{\mathrm{B}}$ and is parallel to the magnetic moment of $\mathrm{Fe}(\mathrm{Co})$. A much larger induced but opposite spin moment in $\mathbf{S}$ layer has been found on Ti atoms : $\sim-0.27(-0.29) \mu_{\mathrm{B}}$.

\section{Electronic properties}

In order to explain the origin of the induced magnetic moments at the interface, we investigated the projected density of states (PDOS) of the free $\mathrm{Fe}(\mathrm{Co})$ slab as well as the $\mathrm{Fe}(\mathrm{Co}) \mid \mathrm{SrTiO}_{3}$ interface compared to the corresponding PDOS in bulk phase of bcc-Fe (fcc-Co) and $\mathrm{SrTiO}_{3}$. As shown in Fig. 3(a), the DOS of the interfacial $\mathrm{Fe}(\mathrm{Co}) 3 d(\mathbf{S}+1)$ (red
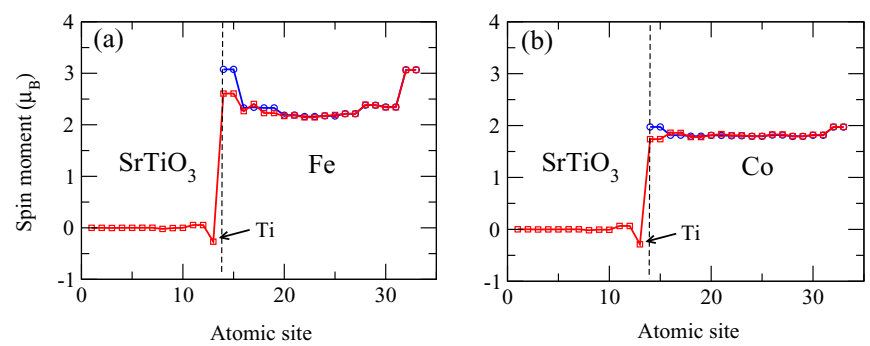

FIG. 2. Layer-resolved magnetic spin moment (in $\mu_{\mathrm{B}}$ ) at $\mathrm{Fe} \mid \mathrm{SrTiO}_{3}(001)$ (a) and $\mathrm{Co} \mid \mathrm{SrTiO}_{3}(001)$ (b) interfaces. Blue circles and red squares correspond to free slab and slab on $\mathrm{SrTiO}_{3}$ substrate, respectively. 
$\mathrm{Fe}$
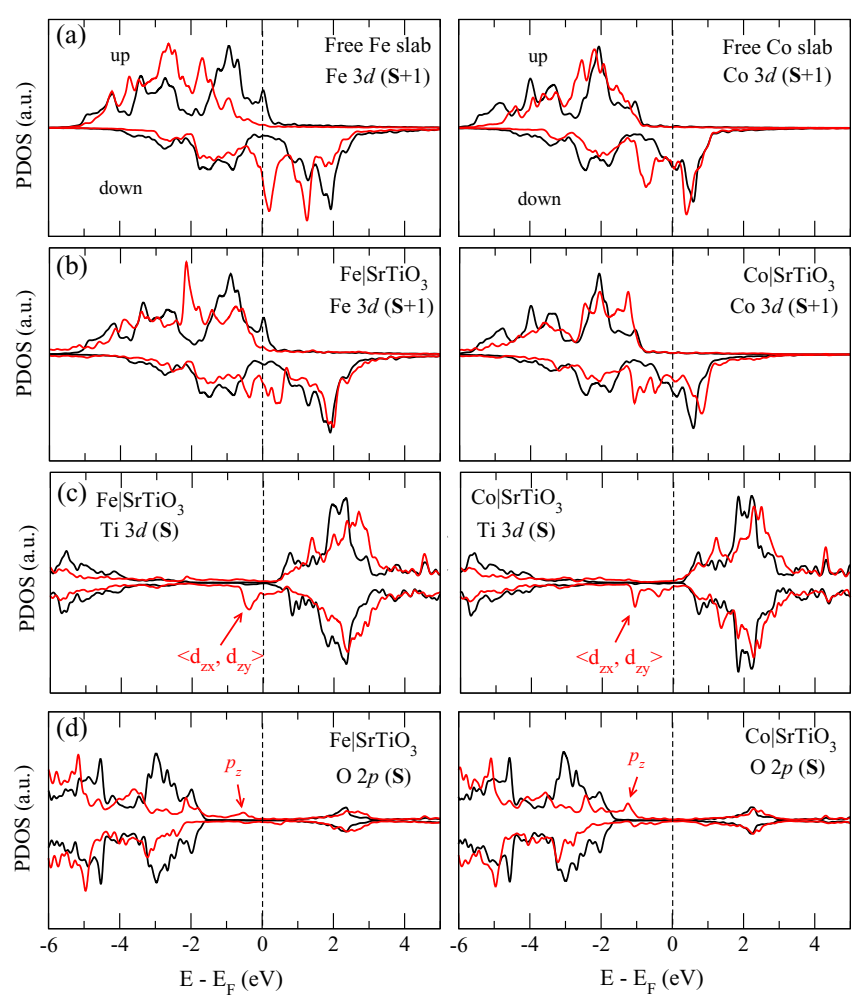

FIG. 3. In red lines (a) free Fe slab: Scalar-relativistic projected density of states (PDOS) of the surface Fe $3 d$ orbitals in layer $\mathbf{S}+1$; (b) $\mathrm{Fe} \mid \mathrm{SrTiO}_{3}(001)$ : PDOS of Fe $3 d$ orbitals in layer $\mathbf{S}+1$, (c) $\mathrm{Ti}$ $3 d$, and (d) O $2 p$ orbitals in layer $\mathbf{S}$. The DOS of atoms in the central monolayer of Fe slab (a), (b) or (c), (d) $\mathrm{TiO}_{2}$ in layer $\mathbf{S}-2$ are plotted as black lines. Positive and negative PDOS are for spin up and spin down channels, respectively. The vertical dashed lines indicate the Fermi level $\left(E_{\mathrm{F}}\right)$. It is the same for Co as presented in the right panels.

line) for free slab differs from the DOS of the bulk $\mathrm{Fe}(\mathrm{Co}) 3 d$ $(\mathbf{S}+5)$ (black line) as a result of the reduced coordination. A significant minority spin states at $\sim 0.1$ and $0.7 \mathrm{eV}(-0.4$ and $0.2 \mathrm{eV}$ ) with respect to the Fermi level has been found for $\mathrm{Fe}(\mathrm{Co})$ at the interface. These states are the origin of the increase of spin moment for the surface atom as shown in Fig. 2.

Figures 3(b)-3(d) show the PDOS of $\mathrm{Fe}(\mathrm{Co}) 3 d(\mathbf{S}+1)$, $\mathrm{Ti} 3 d(\mathbf{S})$ and $\mathrm{O} 2 p(\mathbf{S})$ orbitals at $\mathrm{Fe}(\mathrm{Co}) \mid \mathrm{SrTiO}_{3}$ interface, indicating the presence of hybridizations between the orbitals. It is well known that the degree of hybridization at the interface depends on the strength of the orbital overlap and inversely on the energy separation between them. Although there is a direct atomic bonding between the interfacial $\mathrm{Fe}(\mathrm{Co})$ and $\mathrm{O}$ atoms, the induced magnetic moment on the $\mathrm{O}$ atom was found to be relatively small $\left(\sim 0.05 \mu_{\mathrm{B}}\right)$. This is due to the fact that $\mathrm{O} 2 p$ (S) orbitals lie well below the Fermi level and, therefore, have a small overlap with the $\mathrm{Fe}(\mathrm{Co}) 3 d$ states. However, the Ti $3 d$ orbitals that are centered at about $2 \mathrm{eV}$ above the Fermi level [the black lines in Fig. 3(c)] have a strong hybridization with the minority-spin $\mathrm{Fe}(\mathrm{Co}) 3 d$ orbitals which have a significant weight at these energies [the black lines in Fig. 3(b)]. The most important consequence of this hybridization is the formation of the hybridized states in the interval of energies $[-0.5,+0.5] \mathrm{eV}$ and $[-1,+1] \mathrm{eV}$ for $\mathrm{Fe}$ and $\mathrm{Co}$, respectively. As shown in Fig. 3(c), the DOS of the Ti $3 d \mathbf{S}$ layer at the $\mathrm{Fe}(\mathrm{Co}) \mid \mathrm{SrTiO}_{3}$ interface, the minority-spin states which originates from the $d_{z x}$ and $d_{z y}$ orbitals at $\sim-0.5 \mathrm{eV}$ (the two peaks at $-1 \mathrm{eV}$ and $-0.5 \mathrm{eV}$ ) are occupied, whereas the corresponding majorityspin states are found at $\sim+1.5 \mathrm{eV}$ (the two peaks at $+0.5 \mathrm{eV}$ and $+1 \mathrm{eV}$ ) are unoccupied. This leads to an induced magnetic moment of -0.27 and $-0.29 \mu_{\mathrm{B}}$ on the $\mathrm{Ti}(\mathbf{S})$ for $\mathrm{Fe}$ and $\mathrm{Co}$ based interfaces, respectively.

\section{Local analysis of MCA}

We now investigate the MCA of the $\mathrm{Fe}(\mathrm{Co}) \mid \mathrm{SrTiO}_{3}$ interface. The MCA is calculated as band energy difference between the spin quantization axes perpendicular and parallel to the slab surface, explicitly, $\mathrm{MCA}=E_{\perp}^{\text {band }}-E_{\|}^{\text {band }}$, and for the sake of simplicity we have chosen the most symmetric in plane orientation. By definition a positive (negative) sign in MCA means in-plane (out-of-plane) magnetization axis. It should be noted that the full relativistic Hamiltonian including spin-orbit coupling is given in a basis of total angular momentum eigenstates $\mid j, m_{j}>$ with $j=l \pm \frac{1}{2}$. Although the $\left(l, m_{l}, m_{s}\right)$ is not a well defined quantum number for the full relativistic calculations, the MCA can still be projected into different orbitals and spins by using local density of states. Since the spin-orbit coupling in $3 d$-electron systems is relatively small, this approximate decomposition introduces a negligible numerical inaccuracy.

As shown in Figs. 4(a) and 4(b), we have calculated the atom-resolved MCA of the $\mathrm{Fe}(\mathrm{Co}) \mid \mathrm{SrTiO}_{3}$ system (red squares) and compared it with the free $\mathrm{Fe}(\mathrm{Co})$ slab (blue circles) containing 10 atomic layers (but relaxed in presence of the substrate). For free $\mathrm{Fe}(\mathrm{Co})$ slab, the total MCA reaches $\sim-0.49$ (1.60) $\mathrm{meV}$ per unit-cell favoring an out-of-plane (in-plane) axis of magnetization. When the $\mathrm{Fe}(\mathrm{Co})$ slab is in contact with $\mathrm{SrTiO}_{3}$ substrate, the axis of magnetization is preserved but the total MCA is reduced to $\sim-0.38(1.02) \mathrm{meV}$.

From the atom-resolved MCA, one finds that the MCA curves for free slabs are not symmetrical, particularly pronounced for Co, due to (asymmetrical) relaxation effect. The main contribution to MCA is located in the vicinity of the interface, from $\mathbf{S}$ layer to $\mathbf{S}+3$ layer, marked as a vertical dotted line in Figs. 4(a) and 4(b), and it converges to the expected bulk value in the center of the slab $(\mathbf{S}+5$ layer $)$. Interestingly, at the interface, in comparison with free $\mathrm{Fe}(\mathrm{Co})$ slab it appears that the contact with $\mathrm{SrTiO}_{3}$ strongly favors in-plane and out-of-plane for $\mathrm{Fe}$ and $\mathrm{Co}$, respectively.

For $\mathrm{Fe}(\mathbf{S}+1)$, upon adsorption on $\mathrm{SrTiO}_{3}$, the MCA decreases from $\sim-0.15$ to $\sim-0.06 \mathrm{meV} /$ atom and the out-of-plane magnetization remains. However, in the case of $\mathrm{Co}(\mathbf{S}+1)$, the MCA abruptly changes from $\sim 0.22$ to $\sim-0.25 \mathrm{meV} /$ atom exhibiting magnetization reversal from in-plane to out-of-plane at the same time. For $\mathbf{S}+2$ layer, we find a sign change of MCA between free slab and slab on $\mathrm{SrTiO}_{3}$ for both elements, with the MCA difference of $\sim 0.04 \mathrm{meV} /$ atom and $\sim 0.15 \mathrm{meV} /$ atom for $\mathrm{Fe}$ and $\mathrm{Co}$, respectively. For $\mathbf{S}+3$ layer, the $\mathrm{MCA}$ enhances slightly ( $\sim 0.05 \mathrm{meV} /$ atom) in-plane MCA when depositing slabs on 
$\mathrm{Fe}$
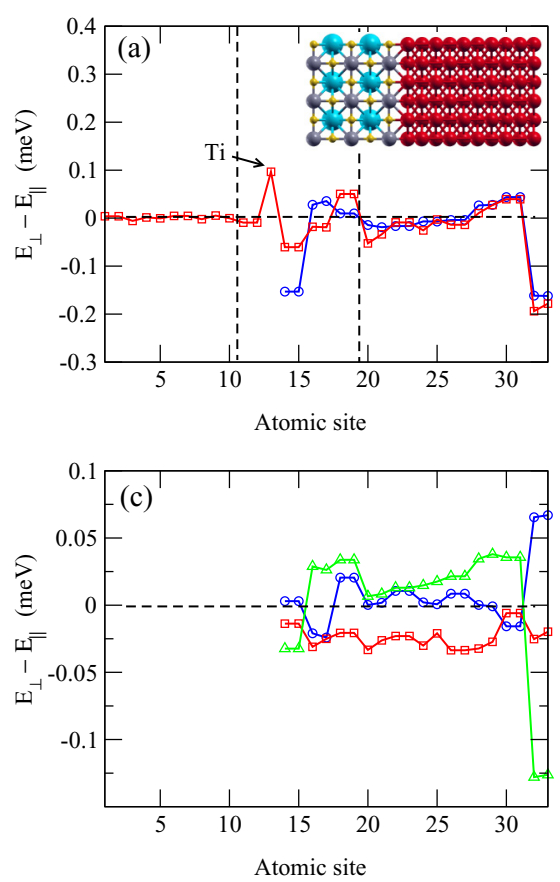

Co
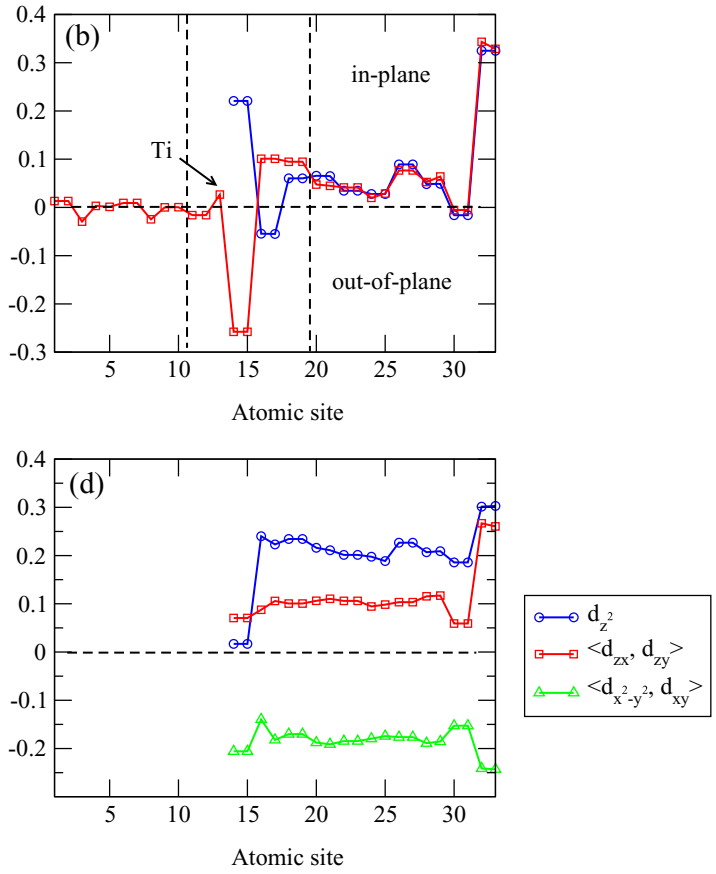

FIG. 4. Atom-resolved $\mathrm{MCA}$ at $\mathrm{Fe} \mid \mathrm{SrTiO}_{3}$ (a) and $\mathrm{Co} \mid \mathrm{SrTiO}_{3}$ (b) interfaces, blue circles and red squares correspond to free slab and slab on a $\mathrm{SrTiO}_{3}$ substrate, respectively. $d$-orbitals-resolved MCA for $\mathrm{Fe}$ (c) and $\mathrm{Co}$ (d) slabs on $\mathrm{SrTiO}_{3}$, we plot only the part of ferromagnetic slabs. Due to symmetry, contributions from different orbitals in $\left(d_{z x}, d_{z y}\right)$ and $\left(d_{x^{2}-y^{2}}, d_{x y}\right)$ pairs are very similar so that their averaged values are presented for simplicity. Note that positive and negative MCA represent in-plane and out-of-plane magnetization, respectively.

$\mathrm{SrTiO}_{3}$ for both elements. Furthermore, the Ti atom in $\mathbf{S}$ layer [indicated by arrows in Figs. 4(a) and 4(b)] presents a rather large in-plane MCA of $\sim 0.1 \mathrm{meV} /$ atom and a much smaller in-plane $\mathrm{MCA}$ of $\sim 0.03 \mathrm{meV} /$ atom for $\mathrm{Fe}$ and Co-based interfaces, respectively. As a result, for free slabs, the MCA values from $\mathbf{S}+1$ layer to $\mathbf{S}+3$ layer sum up to the total value of $\sim-0.22 \mathrm{meV}$ (out-of-plane) and $0.45 \mathrm{meV}$ (in-plane) for $\mathrm{Fe}$ and $\mathrm{Co}$. However, when the slabs are supported on $\mathrm{SrTiO}_{3}$, the overall out-of-plane MCA in the vicinity of the surface (here, the $\mathbf{S}$ layer is also taken into account) is almost quenched for $\mathrm{Fe}$ by $\sim 0 \mathrm{meV}$, and in the case of $\mathrm{Co}$, a spin reorientation from in-plane to out-of-plane magnetization has been found with a MCA value of $\sim-0.10 \mathrm{meV}$.

In order to understand the origin of this difference in MCA between free $\mathrm{Fe}(\mathrm{Co})$ slab and $\mathrm{Fe}(\mathrm{Co}) \mid \mathrm{SrTiO}_{3}$ system, we investigated the $d$-orbitals-resolved MCA of the $\mathrm{Fe}(\mathrm{Co})$ atom as shown in Figs. 4(c) and 4(d). Here, due to symmetry, the contributions to MCA from $\left(d_{z x}, d_{z y}\right)$ and $\left(d_{x^{2}-y^{2}}, d_{x y}\right)$ pairs are almost equal, therefore, their averaged values are presented for simplicity.

In the case of $\mathrm{Fe}$, we notice that going from the free $\mathrm{Fe}$ slab to the $\mathrm{Fe} \mid \mathrm{SrTiO}_{3}$ system, the MCA of the $d_{z^{2}}$ (in-plane magnetization) and $\left(d_{x^{2}-y^{2}}, d_{x y}\right)$ (out-of-plane magnetization) orbitals decreases in magnitude, while the MCA of $\left(d_{z x}, d_{z y}\right)$ orbitals is almost not affected. In addition, quantitatively, the reduction of MCA is larger for $\left(d_{x^{2}-y^{2}}, d_{x y}\right)$ than for $d_{z^{2}}$ due to stronger hybridization between $\left(\mathrm{Fe}-d_{x^{2}-y^{2}, x y}\right.$, Ti- $\left.d_{z x, z y}\right)$ orbitals than between $\left(\mathrm{Fe}-d_{z^{2}}, \mathrm{O}-p_{z}\right)$ orbitals. This is attributed to the fact that, shown in Fig. 3, close to the Fermi level, the shape of the electron density for $\mathrm{O}$ and Ti suggest that this density has a $p_{z}$ character and $d_{z x}\left(d_{z y}\right)$ character, respectively. Moreover, the strong in-plane MCA in $\mathrm{Ti}(\mathbf{S})$ layer originates from the Ti- $d_{z x, z y}$ orbitals since there is a significant weight close to Fermi level of minority-spin (Ti- $\left.d_{z x, z y}\right)$ orbitals [see Fig. 3(c) left panel]. As a result, the MCA at the interface appears to almost quench the out-of-plane magnetization when the $\mathrm{Fe}$ slab is deposited on $\mathrm{SrTiO}_{3}$. Moreover, if we sum over the contribution of the first three layers of Fe slab at the interface, we found that $d_{z x}, d_{z y}$ orbitals tend to maintain the out-of-plane MCA while $d_{x^{2}-y^{2}, x y}$ orbitals tend to favor the in-plane MCA. A similar result has also been reported in Ref. [24] in $\mathrm{Fe} \mid \mathrm{MgO}$ magnetic tunnel junctions.

In the case of $\mathrm{Co}$, we find that the hybridization between $p_{z}$ orbitals of $\mathrm{O}$ and $d_{z^{2}}$ (and, to a slightly lesser extent with $d_{z x, z y}$ ) of Co plays a crucial role to decrease in-plane MCA of the free Co slab. On the other hand, the MCA from in-plane $\left(d_{x^{2}-y^{2}, x y}\right)$ orbitals of Co is less affected due to rather small minority-spin states of (Ti- $\left.d_{z x, z y}\right)$ close to the Fermi level [see Fig. 3 (c) right panel]. This leads to induce an inverse spin orientation transition from in-plane to out-of-plane in $\mathrm{Co} \mid \mathrm{SrTiO}_{3}$ system. A similar result has also been reported in Ref. [25] at $\mathrm{C}_{60} \mid \mathrm{Co}$ interface.

Finally, let us mention that another important contribution to the total magnetic anisotropy should be considered: The so-called shape anisotropy (SA), a relativistic correction that originates from the Breit interaction [26]. Unlike the MCA, the SA is a long-range interaction, and it has a very little dependence on symmetry, crystallographic structure, or local atomic environment. It basically only depends on the shape, volume, and magnetization of the sample. Numerically we 


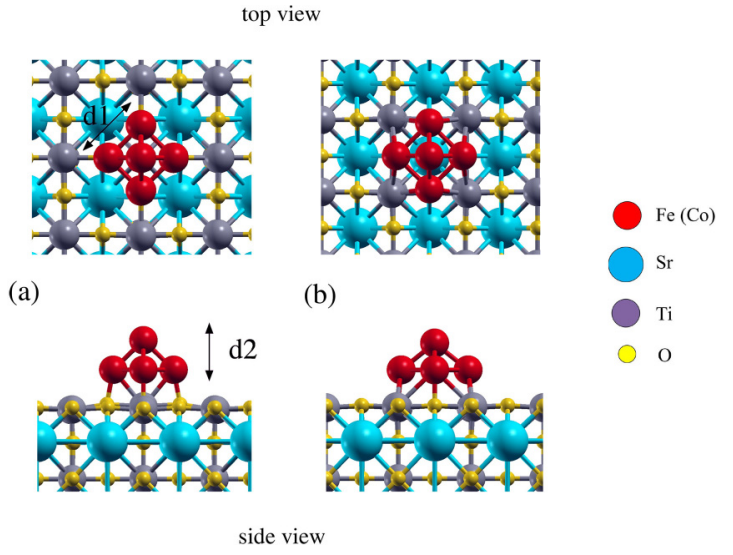

FIG. 5. Top (upper panels) and side (lower panels) views of the optimized geometries of $\mathrm{Fe}$ and Co cluster absorbed on $\mathrm{TiO}_{2}$ terminated $\mathrm{SrTiO}_{3}(001)$. Two different adsorption configurations are presented in (a) and (b), the latter one (hollow geometry) is the most stable configuration for both $\mathrm{Fe}$ and Co clusters. The bond length $d_{1}$ between base atoms and the vertical distance $d_{2}$ between base and top atoms are also indicated.

have calculated the SA by summing over all pairs of magnetic dipole-dipole interaction. For a ten layer free $\mathrm{Fe}(\mathrm{Co})$ slab (containing 20 atoms per surface unit cell), we found a shape anisotropy of $\sim 3.23$ (1.91) meV per unit-cell favoring an in-plane axis of magnetization. $\mathrm{For} \mathrm{Fe}(\mathrm{Co}) \mid \mathrm{SrTiO}_{3}$ interface, it is slightly reduced to $\sim 3.08(1.88) \mathrm{meV}$. This small reduction is due to a decrease of the interface magnetization. For thinner layers the SA will decrease proportionally to the thickness of the sample: Typically the SA of a five layer slab will be divided by two while the MCA will be much less affected.

\section{B. Fe and Co clusters on $\mathrm{SrTiO}_{3}$}

We now investigate the electronic and magnetic properties of $\mathrm{Fe}$ and $\mathrm{Co}$ clusters deposited on $\mathrm{SrTiO}_{3}$ surface. As shown in Fig. 5, two geometries are examined, namely top (a) and hollow (b) adsorption sites. The base atoms of $\mathrm{Fe}(\mathrm{Co})$ clusters are always on top of $\mathrm{O}$ atom for both geometries however the apex atom is either on top of a Ti atom (top geometry) or of an underneath Sr atom (hollow geometry). We found that a hollow adsorption site is more energetically stable for both elements, with an energy difference of $\sim 0.65 \mathrm{eV}$ and $\sim 0.88 \mathrm{eV}$ for $\mathrm{Fe}$ and $\mathrm{Co}$, respectively. In the following, we concentrate on the lowest energy configuration.

The strength of the cluster- $\mathrm{SrTiO}_{3}$ interaction can be quantified by calculating the binding energy via the energy difference:

$$
E_{\mathrm{b}}=E[\text { cluster }]+E\left[\mathrm{SrTiO}_{3}\right]-E\left[\text { cluster }-\mathrm{SrTiO}_{3}\right]
$$

where $\mathrm{E}$ [cluster], $\mathrm{E}\left[\mathrm{SrTiO}_{3}\right]$, and $\mathrm{E}\left[\right.$ cluster $\left.-\mathrm{SrTiO}_{3}\right]$ are the total energy of the free cluster, the free $\mathrm{SrTiO}_{3}$ substrate, and the cluster- $\mathrm{SrTiO}_{3}$ system, respectively. The calculated binding energy was found to be $\sim 4.23$ (4.58) eV for $\mathrm{Fe}(\mathrm{Co})$ cluster on $\mathrm{SrTiO}_{3}$ substrate, showing strong chemisorption mechanism (see Table I).

Compared to free $\mathrm{Fe}$ cluster, the $\mathrm{Fe}-\mathrm{Fe}$ distance in basal plane $\left(d_{1}\right)$ is elongated from $2.31 \AA$ to $2.55 \AA$ while the $\mathrm{Fe}-\mathrm{Fe}$
TABLE I. Binding energies $\left(E_{\mathrm{b}}\right)$, atomic bonds, total/total absolute spin moments $\left(M_{\mathrm{s}}^{\text {tot }} /\left|M_{\mathrm{s}}^{\text {tot }}\right|\right)$, spin moment of base $\left(M_{\mathrm{s}}^{\text {base }}\right)$, and top $\left(M_{\mathrm{s}}^{\text {top }}\right)$ atoms of the free clusters and clusters deposited on $\mathrm{SrTiO}_{3}$ for the lowest energy configuration (hollow geometry).

\begin{tabular}{lccccc}
\hline \hline & \multicolumn{2}{c}{$\mathrm{Fe}$} & & \multicolumn{2}{c}{$\mathrm{Co}$} \\
\cline { 2 - 3 } \cline { 5 - 6 } & $\begin{array}{c}\text { Free } \\
\text { cluster }\end{array}$ & $\begin{array}{c}\text { Cluster } \\
\text { on } \mathrm{SrTiO}_{3}\end{array}$ & & $\begin{array}{c}\text { Free } \\
\text { cluster }\end{array}$ & $\begin{array}{c}\text { Cluster } \\
\text { on } \mathrm{SrTiO}_{3}\end{array}$ \\
\hline$E_{\mathrm{b}}(\mathrm{eV})$ & & 4.23 & & 4.58 \\
$d_{1}(\AA)$ & 2.31 & 2.55 & & 2.17 & 2.20 \\
$d_{2}(\AA)$ & 1.73 & 1.45 & & 1.80 & 1.74 \\
$M_{\mathrm{s}}^{\text {tot }}\left(\mu_{\mathrm{B}}\right)$ & 18.00 & 16.63 & & 13.00 & 7.67 \\
$\left|M_{\mathrm{s}}^{\text {tot }}\right|\left(\mu_{\mathrm{B}}\right)$ & 18.34 & 17.96 & & 13.41 & 11.06 \\
$M_{\mathrm{s}}^{\text {base }}\left(\mu_{\mathrm{B}}\right)$ & 3.62 & 3.33 & & 2.54 & 1.75 \\
$M_{\mathrm{s}}^{\text {top }}\left(\mu_{\mathrm{B}}\right)$ & 3.58 & 3.32 & & 2.84 & 1.57 \\
\hline \hline
\end{tabular}

distance in vertical distance from apex to basal plane $\left(d_{2}\right)$ is compressed from $1.73 \AA$ to $1.45 \AA$ (see Table I). However, in the case of $\mathrm{Co}$, the geometry optimization of $\mathrm{Co}_{5}-\mathrm{SrTiO}_{3}$ results in a rather small (negligible) distortion compared to its free $\mathrm{Co}_{5}$ cluster. In addition, the bond length between $\mathrm{Fe}(\mathrm{Co})$ and $\mathrm{O}$ is $\sim 2 \AA$.

\section{Magnetic spin moment}

We next investigated the local magnetic spin moment. In Table I, the local spin moments for both free clusters and the clusters on $\mathrm{SrTiO}_{3}$ are given. The binding between $\mathrm{Fe}(\mathrm{Co})$ and $\mathrm{O}$ atoms reduces the total spin moment from $18.00 \mu_{\mathrm{B}}$ (free $\mathrm{Fe}_{5}$ ) to $16.63 \mu_{\mathrm{B}}$ and from $13.00 \mu_{\mathrm{B}}$ (free $\mathrm{Co}_{5}$ ) to $7.67 \mu_{\mathrm{B}}$ for the deposited clusters. We also calculated the absolute total spin moment $\left|M_{\mathrm{s}}^{\text {tot }}\right|$ and compared to corresponding total spin moment $M_{\mathrm{s}}^{\text {tot }}$. Interestingly, a substantial difference of $\sim 3.4 \mu_{\mathrm{B}}$ has been found between $\left|M_{\mathrm{s}}^{\text {tot }}\right|$ and $M_{\mathrm{s}}^{\text {tot }}$ for $\mathrm{Co}_{5}-\mathrm{SrTiO}_{3}$. In order to understand the origin of this difference, we plot in Fig. 6 the real-space distribution of magnetic spin moment of Co cluster on $\mathrm{SrTiO}_{3}$. Note that the red (blue) corresponds to positive (negative) spin moment. We can see clearly the negative magnetic moment is mainly localized on $\mathrm{Ti}$ atoms at the interface and around the Co top atom of cluster. However, for Fe cluster, the positive spin moment is very localized on the $\mathrm{Fe}$ atoms and the negative part is negligible.
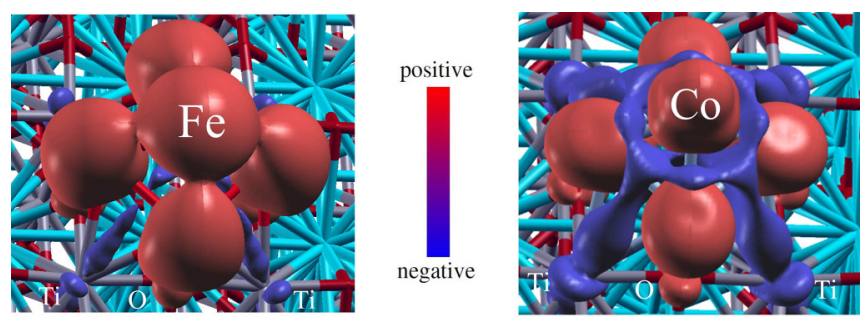

FIG. 6. Real-space distribution of magnetic spin moment of $\mathrm{Fe}$ (left) and $\mathrm{Co}$ (right) cluster on $\mathrm{SrTiO}_{3}$. Note that red (blue) corresponds to positive (negative) spin moment. The nonnegligible negative part of spin moment has been found around the Ti atoms at the interface and the Co top atom of cluster. 

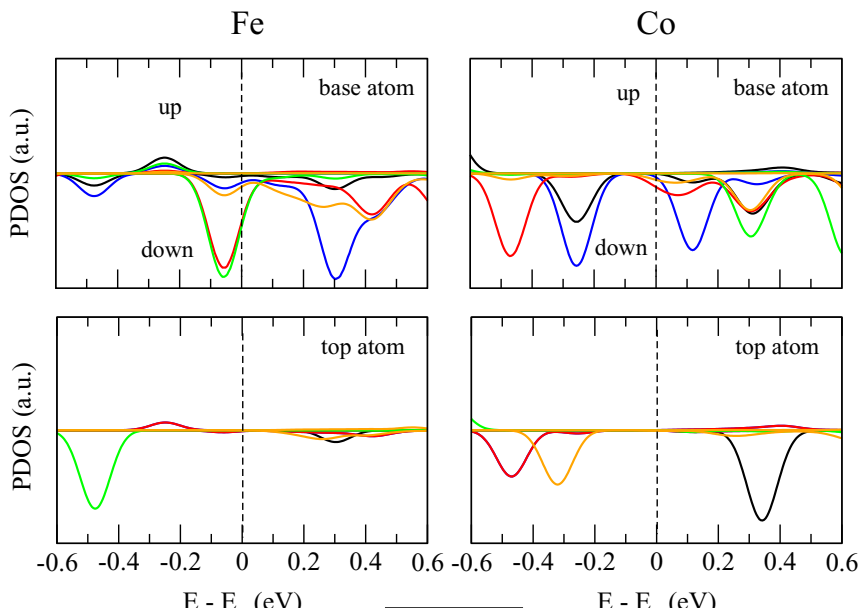

$\mathrm{E}-\mathrm{E}_{\mathrm{F}}(\mathrm{eV})$

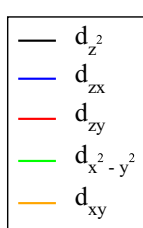

FIG. 7. Scalar-relativistic $d$-orbitals projected density of states (PDOS) for $\mathrm{Fe}(\mathrm{Co})$ base atom (a) and top atom (b) of the cluster absorbed on $\mathrm{SrTiO}_{3}$. Positive and negative PDOS are for spin up and spin down channels, respectively. The vertical dashed lines mark the Fermi level $\left(E_{\mathrm{F}}\right)$.

\section{Electronic structure properties}

To gain more insight into the electronic structure of $\mathrm{Fe}_{5}-\mathrm{SrTiO}_{3}$ and $\mathrm{Co}_{5}-\mathrm{SrTiO}_{3}$, we plot the scalar-relativistic PDOS on $d$ orbitals of $\mathrm{Fe}(\mathrm{Co})$ base atom and top atom of the cluster in Figs. 7(a) and 7(b). For the base atom of both clusters, the density of majority states is almost completely occupied (situated below $-0.6 \mathrm{eV}$ ) and negligibly small around the Fermi level, while the density of minority states is partially occupied. Around the Fermi level, there is a higher density of $\left(d_{x^{2}-y^{2}}, d_{x y}, d_{z y}\right)$ states for Fe while the most dominant states are the out-of-plane $d$ orbitals for Co, namely $\left(d_{z^{2}}, d_{z x}, d_{z y}\right)$ orbitals. For top atom, in the interval of energies $[-0.25$, $+0.25] \mathrm{eV}$, the density of states for both majority and minority spins is negligibly small for both clusters.

\section{Local analysis of MCA}

The MCA is calculated by the formula $\mathrm{MCA}=E_{z}^{\text {band }}-$ $E_{x^{\prime}}^{\text {band }}$ using as usual the magnetic force theorem. The MCA in the $x y$ plane is found to be extremely small. We have chosen the most symmetric in-plane direction $x^{\prime}$ (see Fig. 8) which has an azimuthal angle of $\phi=45^{\circ}$ with respect to $x$. Due to symmetry, this definition gives us almost similar contribution for each pair of $\left(d_{z x}, d_{z y}\right)$ and of $\left(d_{x^{2}-y^{2}}, d_{x y}\right) \mathrm{Fe}(\mathrm{Co})$ orbitals, therefore, their averaged values are presented for the sake of simplicity.

In Figs. 8(a) and 8(b) the local decomposition of MCA with different atomic sites as well as with different $d$ orbitals is presented for $\mathrm{Fe}_{5}-\mathrm{SrTiO}_{3}$ and $\mathrm{Co}_{5}-\mathrm{SrTiO}_{3}$, respectively. Note that only the contributions of clusters is shown. Interestingly, we find the opposite behavior of MCA for $\mathrm{Fe}$ and $\mathrm{Co}$ clusters deposited on $\mathrm{SrTiO}_{3}$. The easy axis of magnetization is directed along out-of-plane for Fe cluster with a total MCA of $\sim-5.08 \mathrm{meV}$, on the contrary it is in-plane for Co with a total MCA of $\sim 4.72 \mathrm{meV}$. For both elements, the atomically resolved MCA (black lines) reveals that the MCA is mainly dominated by the base atoms (numbered as $1 \sim 4$ ) and a relatively much smaller contribution from the top atom (numbered as 5). The value of MCA per atom is as large as $\sim-1.22(1.08) \mathrm{meV} /$ atom for base atom and $\sim-0.18(0.38) \mathrm{meV} /$ atom for the top atom of $\mathrm{Fe}(\mathrm{Co})$ cluster. It is also interesting to note that the MCA mainly originates from the $d$ orbitals of the cluster extending in-plane for $\mathrm{Fe}$, namely $\left(d_{x^{2}-y^{2}}, d_{x y}\right)$ orbitals, and out-of-plane for Co, namely, $\left(d_{z^{2}}, d_{z x}, d_{z y}\right)$.

Finally in Figs. 8(c) and 8(d), we present the real-space distribution of $\mathrm{MCA}$ for $\mathrm{Fe}_{5}-\mathrm{SrTiO}_{3}$ and $\mathrm{Co}_{5}-\mathrm{SrTiO}_{3}$. The red colors represent in-plane magnetization direction, whereas the blue colors are out-of-plane easy axis. We can clearly se that the MCA mainly originates from the base atoms for both clusters, and for $\mathrm{Fe}(\mathrm{Co})$ the MCA originates from $d$ orbitals of the cluster extending in-plane (out-of-plane). In addition, due to hybridization between the states of $\mathrm{TiO}_{2}$ surface and $d$ orbitals of the cluster, the $\mathrm{Ti}$ and $\mathrm{O}$ atoms close to the cluster gives a rather small contribution to MCA. For Fe, Ti atom slightly favors the in-plane easy axis and the easy axis of $\mathrm{O}$ atom is out-of-plane. In the case of $\mathrm{Co}$, both $\mathrm{Ti}$ and $\mathrm{O}$ atoms around the cluster favor to in-plane magnetization direction. Contrary to the case of thin films the contribution of shape anisotropy to the total magnetic anisotropy is negligible for these very small objects. As a consequence, we predict that the Fe nanocrystals should be magnetically stable and are thus good potential candidates for magnetic storage devices.

\section{MCA analysis from perturbation theory}

Let us consider the perturbation of the total energy due to the spin-orbit coupling (SOC) Hamiltonian $H_{\text {SOC }}$ [27-31]. Since the first-order term vanishes the second order perturbation term $\Delta E^{(2)}$ of the total energy has to be evaluated:

$$
\Delta E^{(2)}=-\sum_{\substack{n \sigma \mathrm{occ} \\ n^{\prime} \sigma^{\prime} \text { unocc }}} \frac{\left|\left\langle n \sigma\left|H_{\mathrm{SOC}}\right| n^{\prime} \sigma^{\prime}\right\rangle\right|^{2}}{E_{n^{\prime} \sigma^{\prime}}-E_{n \sigma}}
$$

where $|n \sigma\rangle\left(\left|n^{\prime} \sigma^{\prime}\right\rangle\right)$ is an unperturbed occupied (unoccupied) state of energy $E_{n \sigma}\left(E_{n^{\prime} \sigma^{\prime}}\right), n$ denotes the index of the state and $\sigma$ its spin (which is still a good quantum number for the unperturbed state). Writing the eigenstates in an orthogonal basis of real atomic spin orbitals $\lambda \sigma$ centered at each atomic site $i$, one can derive a rather cumbersome equation written explicitly in the Appendix of Ref. [29] (Eq. C.8). However it is possible to drastically simplify Eq. C.8 by retaining only the diagonal terms of the density matrix which leads to the following expression:

$$
\Delta E^{(2)}=A-\xi^{2} \sum_{\lambda \mu}\left|\left\langle\lambda \uparrow\left|H_{\mathrm{SOC}}\right| \mu \uparrow\right\rangle\right|^{2} \sum_{i \sigma \sigma^{\prime}} \sigma \sigma^{\prime} I_{i}\left(\lambda, \mu, \sigma, \sigma^{\prime}\right)
$$

where $A$ is a constant isotropic term and

$$
I_{i}\left(\lambda, \mu, \sigma, \sigma^{\prime}\right)=\int_{-\infty}^{E_{F}} d E \int_{E_{F}}^{\infty} d E^{\prime} \frac{n_{i \lambda \sigma}(E) n_{i \mu \sigma^{\prime}}\left(E^{\prime}\right)}{E^{\prime}-E}
$$



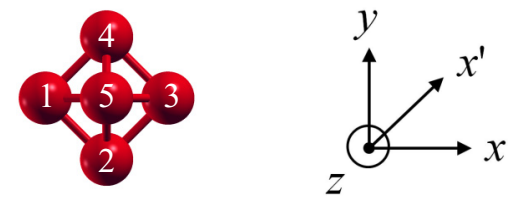

(a)

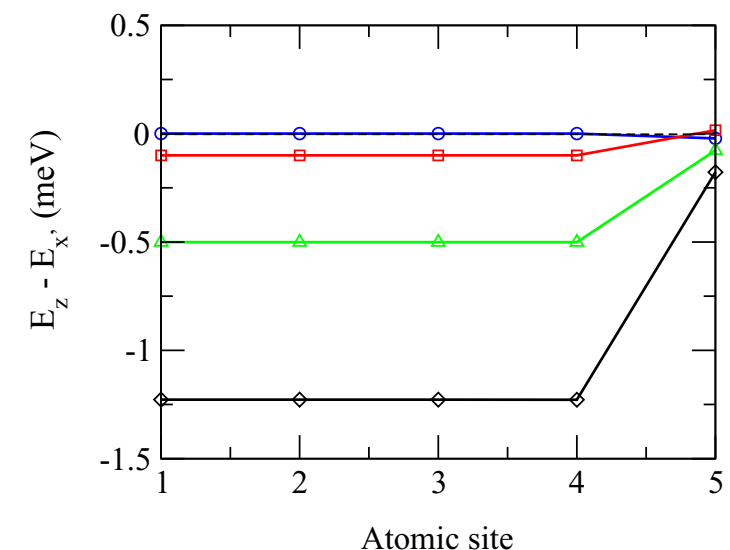

(c)

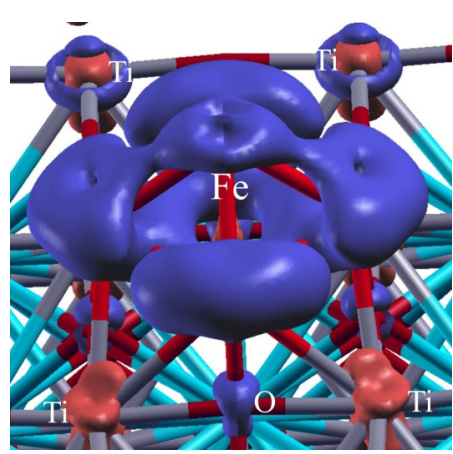

(b)

Co

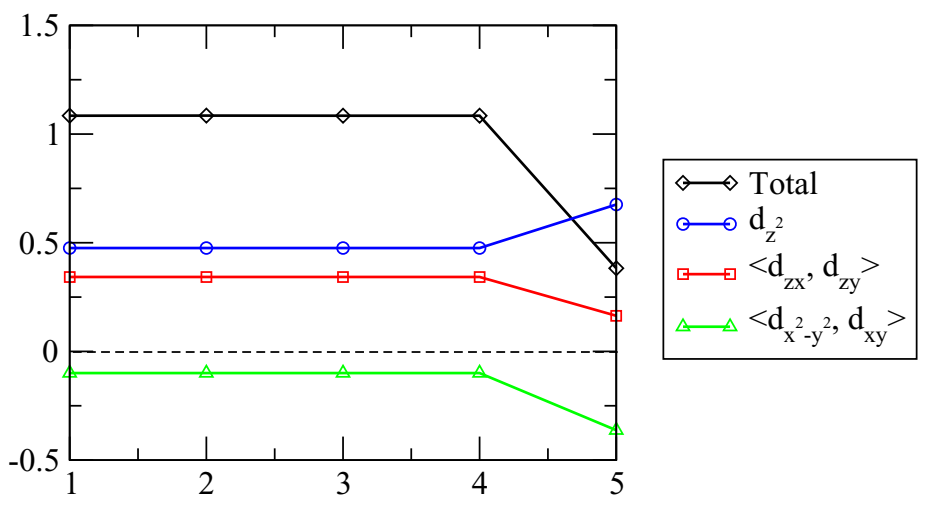

Atomic site

(d)

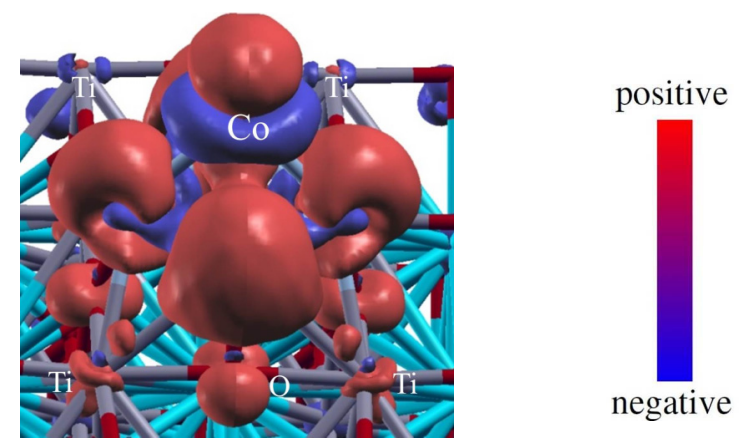

FIG. 8. Atom/ $d$-orbitals-resolved MCA of $\mathrm{Fe}$ (a) and $\mathrm{Co}$ (b) clusters deposited on $\mathrm{SrTiO}_{3}$. Due to symmetry, contributions from different orbitals in $\left(d_{z x}, d_{z y}\right)$ and $\left(d_{x^{2}-y^{2}}, d_{x y}\right)$ pairs are very similar so that their averaged values are presented for simplicity. Clear out-of-plane and in-plane MCA have been found for Fe and Co clusters, respectively. Real-space distribution of MCA for Fe (c) and Co (d) clusters is given. Note that red (blue) colors represent the regions favoring in-plane (out-of-plane) magnetization orientation. The MCA mainly from the base atoms for both clusters, and for $\mathrm{Fe}(\mathrm{Co})$ the MCA originates from $d$-orbitals of the cluster extending in-plane (out-of-plane).

$n_{i \lambda \sigma}(E)\left(n_{i \mu \sigma^{\prime}}\left(E^{\prime}\right)\right)$ being the projected density of states of occupied (unoccupied) states. The dominant terms $I_{i}\left(\lambda, \mu, \sigma, \sigma^{\prime}\right)$ are the ones corresponding to a transition between an occupied and an unoccupied state presenting a high density of states below and above the Fermi level, respectively.

The MCA defined as the difference of energy between the direction $z$ and $x$ can be decomposed in local atomic contributions $\mathrm{MCA}_{i}$ :

$$
\mathrm{MCA}_{i}=\xi^{2} \sum_{\substack{\lambda \sigma \\ \mu \sigma^{\prime}}} \sigma \sigma^{\prime} T_{\lambda, \mu} I_{i}\left(\lambda, \mu, \sigma, \sigma^{\prime}\right)
$$

$T_{\lambda, \mu}$ is the difference of the square of the spin-orbit matrix elements between two orientations of the magnetization $\mathbf{M}$ :

$$
T_{\lambda, \mu}=|\langle\lambda \uparrow|\mathbf{L} \cdot \mathbf{S}| \mu \uparrow\rangle|_{\mathbf{M} \| x}^{2}-|\langle\lambda \uparrow|\mathbf{L} \cdot \mathbf{S}| \mu \uparrow\rangle|_{\mathbf{M} \| z}^{2}
$$

where $\mathbf{L}$ and $\mathbf{S}$ are the angular orbital and spin momentum operators. Since $I_{i}\left(\lambda, \mu, \sigma, \sigma^{\prime}\right)$ is always positive, the sign of the matrix elements $\sigma \sigma^{\prime} T_{\lambda, \mu}$ for a given transition between an occupied state $\lambda \sigma$ and an unoccupied state $\mu \sigma^{\prime}$ will define the sign of the corresponding anisotropy. In practice there are a limited number of transitions and in addition spin-flip transitions are often negligible, therefore in most case $\sigma \sigma^{\prime}=1$.

Let us now apply this perturbation expansion to the case of $\mathrm{Fe}$ and Co clusters on $\mathrm{SrTiO}_{3}$. First, it is clear from the PDOS analysis that the top atom will contribute negligibly to the total MCA. In contrast for both atoms the PDOS of the base atom shows that there are dominantly four occupied-unoccupied transitions that will dominate the MCA. Namely the transition $d_{x^{2}-y^{2}} \rightarrow d_{z x}, d_{x^{2}-y^{2}} \rightarrow d_{x y}, d_{z y} \rightarrow d_{z x}$, and $d_{z y} \rightarrow d_{x y}$ for Fe and $d_{z x} \rightarrow d_{z x}, d_{z x} \rightarrow d_{z y}, d_{z^{2}} \rightarrow d_{z x}$, and $d_{z^{2}} \rightarrow d_{z y}$ for Co. For the explicit matrix elements of $H_{\text {SOC }}$ in the $d$ orbital basis, please see Appendix. From Eq. (A3) it comes out that for Fe two transitions are large with a negative sign $\left(d_{x^{2}-y^{2}} \rightarrow d_{x y} \propto-4, d_{z y} \rightarrow d_{z x} \propto-1\right)$ and two are small with a positive $\operatorname{sign}\left(d_{x^{2}-y^{2}} \rightarrow d_{z x} \propto 1 / 2, d_{z y} \rightarrow d_{x y} \propto 1 / 2\right)$. 
For Co we find the opposite trend: two transitions have a large and positive $\operatorname{sign}\left(d_{z^{2}} \rightarrow d_{z x} \propto 3 / 2, d_{z^{2}} \rightarrow d_{z y} \propto 3 / 2\right)$, one has a negative $\operatorname{sign}\left(d_{z x} \rightarrow d_{z y} \propto-1\right)$ and the last one is diagonal and do not contribute $\left(d_{z x} \rightarrow d_{z x}=0\right)$. Overall this shows that Fe pyramid favors out-of-plane magnetization while Co favors in plane magnetization. The main orbitals involved are $\left(d_{x y}, d_{x^{2}-y^{2}}\right)$ for Fe and $\left(d_{z x}, d_{z y}, d_{z^{2}}\right)$ for Co in agreement with the results presented in Sec. III B 3 .

This type of analysis remains qualitative and applies preferentially to low dimensional systems presenting sharp features in their PDOS. Nevertheless, the arguments put forward are rather general and could be very useful in the design of atomic-scale devices with optimized magnetic anisotropy. Note however, that if the nonsphericity of the Coulomb and exchange interaction [32] starts to play a dominant role in the electronic structure of the system, then orbital polarization effects arise [33,34] and our analysis of the MCA based on a perturbation treatment of the SOC only non longer applies, and more complex scenarii can occur as in the case of the giant magnetic anisotropy of single adatom on $\mathrm{MgO}$ [35].

\section{CONCLUSION}

To conclude, we investigated the electronic properties and MCA of $\mathrm{Fe}$ and $\mathrm{Co}$ slabs and nanoclusters interfaced with $\mathrm{SrTiO}_{3}$ underlayer. Interestingly, a comparative study of $\mathrm{Fe}$ and $\mathrm{Co}$ freestanding slabs with their interface with $\mathrm{SrTiO}_{3}$, revealed a tremendous impact of the latter on the MCA. Namely, the MCA contribution from the interfacial $\mathrm{Fe}$ layer in $\mathrm{Fe} \mid \mathrm{SrTiO}_{3}$ is quenched resulting in the loss of the perpendicular magnetic anisotropy (PMA) while for $\mathrm{Co} \mid \mathrm{SrTiO}_{3}$, the MCA is changed from in-plane to out-of-plane. This is explained by the orbital resolved analysis of hybridizations of $\mathrm{Fe}$ and $\mathrm{Co} d$-orbitals of with those of $\mathrm{Ti}$ and $p_{z}$ orbital of $\mathrm{O}$.

We also find a strong enhancement of out-of-plane and in-plane MCA for small $\mathrm{Fe}$ and Co clusters (containing only five atoms) upon deposition on a $\mathrm{SrTiO}_{3}$ substrate. The hybridization between the substrate and the $d$-orbitals of the cluster extending in-plane for $\mathrm{Fe}$ and out-of-plane for $\mathrm{Co}$ is at the origin of this enhancement of MCA. As a consequence, we predict that the Fe nanocrystals (even rather small) should be magnetically stable and are thus good potential candidates for future magnetic storage applications.

\section{ACKNOWLEDGMENTS}

The research leading to these results has received funding from the European Research Council under the European Unions Seventh Framework Programme (FP7/20072013)/ERC grant agreement No. 259297. This work was performed using HPC computation resources from GENCI[TGCC] (Grant No. 2015097416).

\section{APPENDIX: EXPRESSION OF THE T MATRIX}

The matrix elements of the spin-orbit coupling Hamiltonian in the $d$ orbital basis (ordered as $d_{x y}, d_{z y}, d_{z x}, d_{x^{2}-y^{2}}, d_{z^{2}}$ ) are written explicitly in Appendix A of Ref. [29] for an arbitrary orientation of the magnetization defined by the altitude angle and the azimuth angle $(\theta, \phi)$. If we define the MCA as the total energy difference between a magnetization along $z(\theta=\phi=$ 0 ) and a magnetization along an arbitrary direction $\mathbf{n}(\theta, \phi)$ the corresponding $\mathbf{T}$ matrix reads:

$$
\frac{1}{4}\left[\begin{array}{ccccc}
0 & \sin ^{2} \theta \sin ^{2} \phi & \sin ^{2} \theta \cos ^{2} \phi & -4 \sin ^{2} \theta & 0 \\
\sin ^{2} \theta \sin ^{2} \phi & 0 & -\sin ^{2} \theta & \sin ^{2} \theta \cos ^{2} \phi & 3 \sin ^{2} \theta \cos ^{2} \phi \\
\sin ^{2} \theta \cos ^{2} \phi & -\sin ^{2} \theta & 0 & \sin ^{2} \theta \sin ^{2} \phi & 3 \sin ^{2} \theta \sin ^{2} \phi \\
-4 \sin ^{2} \theta & \sin ^{2} \theta \cos ^{2} \phi & \sin ^{2} \theta \sin ^{2} \phi & 0 & 0 \\
0 & 3 \sin ^{2} \theta \cos ^{2} \phi & 3 \sin ^{2} \theta \sin ^{2} \phi & 0 & 0
\end{array}\right] .
$$

If $\mathbf{n}$ is along $x(\theta=\pi / 2, \phi=0), \mathbf{T}$ takes the form:

$$
\frac{1}{4}\left[\begin{array}{rrrrr}
0 & 0 & 1 & -4 & 0 \\
0 & 0 & -1 & 1 & 3 \\
1 & -1 & 0 & 0 & 0 \\
-4 & 1 & 0 & 0 & 0 \\
0 & 3 & 0 & 0 & 0
\end{array}\right]
$$

while if instead of $x$ we take the more symmetric in plane $x^{\prime} \operatorname{direction}((\theta=\pi / 2, \phi=\pi / 4)$ we find for $\mathbf{T}$ :

$$
\frac{1}{4}\left[\begin{array}{ccccc}
0 & 1 / 2 & 1 / 2 & -4 & 0 \\
1 / 2 & 0 & -1 & 1 / 2 & 3 / 2 \\
1 / 2 & -1 & 0 & 1 / 2 & 3 / 2 \\
-4 & 1 / 2 & 1 / 2 & 0 & 0 \\
0 & 3 / 2 & 3 / 2 & 0 & 0
\end{array}\right]
$$

A positive sign means an easy axis along $\mathbf{n}$ and a negative sign an easy axis along $z$. 
[1] L. E. Nistor, B. Rodmacq, S. Auffret, and B. Dieny, Appl. Phys. Lett. 94, 012512 (2009).

[2] G. Kim, Y. Sakuraba, M. Oogane, Y. Ando, and T. Miyazaki, Appl. Phys. Lett. 92, 172502 (2008).

[3] K. Mizunuma, S. Ikeda, J. H. Park, H. Yamamoto, H. Gan, K. Miura, H. Hasegawa, J. Hayakawa, F. Matsukura, and H. Ohno, Appl. Phys. Lett. 95, 232516 (2009).

[4] B. G. Park, J. Wunderlich, D. A. Williams, S. J. Joo, K. Y. Jung, K. H. Shin, K. Olejník, A. B. Shick, and T. Jungwirth, Phys. Rev. Lett. 100, 087204 (2008).

[5] L. Gao, X. Jiang, S.-H. Yang, J. D. Burton, E. Y. Tsymbal, and S. S. P. Parkin, Phys. Rev. Lett. 99, 226602 (2007).

[6] N. Nakajima, T. Koide, T. Shidara, H. Miyauchi, H. Fukutani, A. Fujimori, K. Iio, T. Katayama, M. Nývlt, and Y. Suzuki, Phys. Rev. Lett. 81, 5229 (1998).

[7] D. Weller, Y. Wu, J. Stöhr, M. G. Samant, B. D. Hermsmeier, and C. Chappert, Phys. Rev. B 49, 12888 (1994).

[8] S. Bornemann, O. Šipr, S. Mankovsky, S. Polesya, J. B. Staunton, W. Wurth, H. Ebert, and J. Minár, Phys. Rev. B 86, 104436 (2012).

[9] S. Monso, B. Rodmacq, S. Auffret, G. Casali, F. Fettar, B. Gilles, B. Dieny, and P. Boyer, Appl. Phys. Lett. 80, 4157 (2002).

[10] S. Ikeda, K. Miura, H. Yamamoto, K. Mizunuma, H. D. Gan, M. Endo, S. Kanai, J. Hayakawa, F. Matsukura, and H. Ohno, Nat. Mater. 9, 721 (2010).

[11] H. X. Yang, M. Chshiev, B. Dieny, J. H. Lee, A. Manchon, and K. H. Shin, Phys. Rev. B 84, 054401 (2011).

[12] I. G. Rau, S. Baumann, S. Rusponi, F. Donati, S. Stepanow, L. Gragnaniello, J. Dreiser, C. Piamonteze, F. Nolting, S. Gangopadhyay et al., Science 344, 988 (2014).

[13] S. Loth, S. Baumann, C. P. Lutz, D. Eigler, and A. J. Heinrich, Science 335, 196 (2012).

[14] A. A. Khajetoorians and J. Wiebe, Science 344, 976 (2014).

[15] D. Li, A. Smogunov, C. Barreteau, F. Ducastelle, and D. Spanjaard, Phys. Rev. B 88, 214413 (2013).

[16] D. Li, C. Barreteau, M. R. Castell, F. Silly, and A. Smogunov, Phys. Rev. B 90, 205409 (2014).
[17] F. Silly and M. R. Castell, Appl. Phys. Lett. 87, 063106 (2005).

[18] P. Giannozzi, S. Baroni, N. Bonini, M. Calandra, R. Car, C. Cavazzoni, D. Ceresoli, G. L. Chiarotti, M. Cococcioni, I. Dabo et al., J. Phys. Condens. Matter 21, 395502 (2009).

[19] J. P. Perdew, K. Burke, and M. Ernzerhof, Phys. Rev. Lett. 77, 3865 (1996).

[20] M. Fechner, S. Ostanin, and I. Mertig, Phys. Rev. B 77, 094112 (2008).

[21] J. M. De Teresa, A. Barthélémy, A. Fert, J. P. Contour, R. Lyonnet, F. Montaigne, P. Seneor, and A. Vaurès, Phys. Rev. Lett. 82, 4288 (1999).

[22] J. M. De Teresa, A. Barthélémy, A. Fert, J. P. Contour, F. Montaigne, and P. Seneor, Science 286, 507 (1999).

[23] I. I. Oleinik, E. Y. Tsymbal, and D. G. Pettifor, Phys. Rev. B 65, 020401 (2001).

[24] A. Hallal, H. X. Yang, B. Dieny, and M. Chshiev, Phys. Rev. B 88, 184423 (2013).

[25] K. Bairagi, A. Bellec, V. Repain, C. Chacon, Y. Girard, Y. Garreau, J. Lagoute, S. Rousset, R. Breitwieser, Y.-C. Hu et al., Phys. Rev. Lett. 114, 247203 (2015).

[26] H. J. F. Jansen, Phys. Rev. B 38, 8022 (1988).

[27] P. Bruno, Phys. Rev. B 39, 865 (1989).

[28] G. H. O. Daalderop, P. J. Kelly, and M. F. H. den Schuurnans, Phys. Rev. B 50, 9989 (1994).

[29] G. Autès, C. Barreteau, D. Spanjaard, and M.-C. Desjonquères, J. Phys. Condens. Matter 18, 6785 (2006).

[30] M. Tsujikawa and T. Oda, Phys. Rev. Lett. 102, 247203 (2009).

[31] F. Gimbert and L. Calmels, Phys. Rev. B 86, 184407 (2012).

[32] V. I. Anisimov, I. V. Solovyev, M. A. Korotin, M. T. Czyżyk, and G. A. Sawatzky, Phys. Rev. B 48, 16929 (1993).

[33] M. C. Desjonquères, C. Barreteau, G. Autès, and D. Spanjaard, Eur. Phys. J. B 55, 23 (2007).

[34] M.-C. Desjonquères, C. Barreteau, G. Autès, and D. Spanjaard, Phys. Rev. B 76, 024412 (2007).

[35] X. Ou, H. Wang, F. Fan, Z. Li, and H. Wu, Phys. Rev. Lett. 115, 257201 (2015). 